\title{
AN INTRINSIC CORRELATION BETWEEN GRB OPTICAL/UV AFTERGLOW BRIGHTNESS AND DECAY RATE
}

\author{
S.R. Oates ${ }^{1}$, M.J. Page ${ }^{1}$, M. De Pasquale ${ }^{2}$, P. Schady ${ }^{3}$, A.A. Breeveld ${ }^{1}$, \\ S.T. Holland ${ }^{4}$, N.P.M. Kuin ${ }^{1}$ and F.E. Marshall ${ }^{5}$
}

\begin{abstract}
We examine 48 Swift/UVOT long Gamma-ray Burst light curves and find a correlation between the logarithmic luminosity at $200 \mathrm{~s}$ and average decay rate determined from $200 \mathrm{~s}$ onwards, with a Spearman rank coefficient of -0.58 at a significance of $99.998 \%(4.2 \sigma)$. We determine the $\log \mathrm{L}_{200 \mathrm{~s}}-\alpha_{>200 \mathrm{~s}}$ correlation to be intrinsic and discuss two possible causes: there is a property of the central engine, outflow or external medium that effects the rate of energy release so that the bright afterglows release their energy more quickly and decay faster than the fainter afterglows; alternatively, the observers viewing angle may produce the correlation, with observers at large viewing angles observing fainter and slower decaying light curves.
\end{abstract}

\section{Introduction}

In 2009, we analyzed a sample of 26 optical long Gamma-Ray Burst (LGRB) light curves (Oates et al. 2009), unearthing a correlation between the observed $v$-band magnitude at $400 \mathrm{~s}$ and the average UVOT light curve decay rate determined from $500 \mathrm{~s}$. In order to have implications on our understanding of LGRBs this correlation must be observed in the rest frame, however due to the small sample size we could not confirm or exclude a correlation. Here we use a larger sample of

1 Mullard Space Science Laboratory, University College London, Holmbury St. Mary, Dorking Surrey, RH5 6NT, UK; e-mail: sro@mssl.ucl.ac.uk

2 University of Nevada, Las Vegas, Department of Physics \& Astronomy, Box 454002, 4505 Maryland Parkway, Las Vegas, NV 89154-4002, USA

3 Max-Planck-Institut für Extraterrestrische Physik, Giessenbachstraße 1, 85748 Garching, Germany

4 Space Telescope Science Center, 3700 San Martin Dr., Baltimore, MD 21218, USA

5 Astrophysics Science Division, Code 660.1, NASA Goddard Space Flight Centre, 8800 Greenbelt Road, Greenbelt, Maryland 20771, USA 


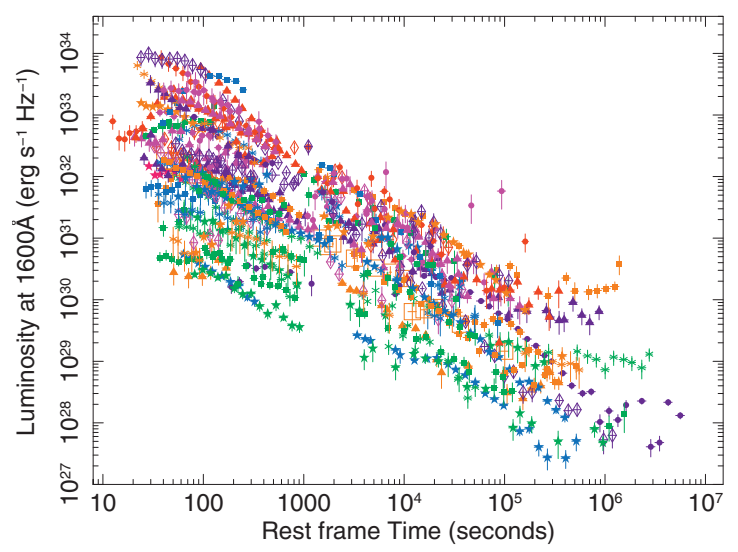

Fig. 1. Optical luminosity light curves of 56 LGRBs at restframe $1600 \AA$ A. For clarity, $3 \sigma$ upper limits are not included.

48 high quality LGRB UVOT light curves to re-examine if there is a correlation between optical/UV afterglow intrinsic brightness and light curve decay rate.

\section{Luminosity light curves}

We selected 69 LGRBs from the second Swift UVOT GRB afterglow catalogue (Roming et al. 2013) using the criteria in Oates et al. (2009): the light curves must have a peak UVOT $v$-band magnitude brighter than 17.89, UVOT must observe from $\leq 400$ to $\geq 10^{5} \mathrm{~s}$ after the BAT trigger and the colour of the afterglows must not evolve significantly with time, so that at no stage should the light curve from a single filter significantly deviate from any other filter light curve when normalized to the $v$ filter. These ensure a high signal-to-noise light curve, covering both early and late times, can be constructed from the UVOT multi-filter observations using the method in Oates et al. (2009). The main steps were to normalize the multifilter light curves to the $v$ filter and then group them using a binsize of $\Delta t / t=0.2$.

Of the 69 GRBs, luminosity light curves could be produced for the 56 that had redshifts and for which host $\mathrm{E}(\mathrm{B}-\mathrm{V})$ values could be determined. For each of the 56 GRBs, the single filter count rate light curves were converted to luminosity at a common restframe wavelength of $1600 \AA$, and were corrected for both Galactic and host extinction.

\section{Results}

The luminosity light curves at $1600 \AA$, given in Figure 1 with units of $\mathrm{erg} \mathrm{s}^{-1} \mathrm{~Hz}^{-1}$, are clustered in a single group, with the largest range in luminosity at the earliest epochs, which becomes narrower as the light curves decay. This suggests that the most luminous GRBs decay the quickest, while the less luminous GRBs decay 


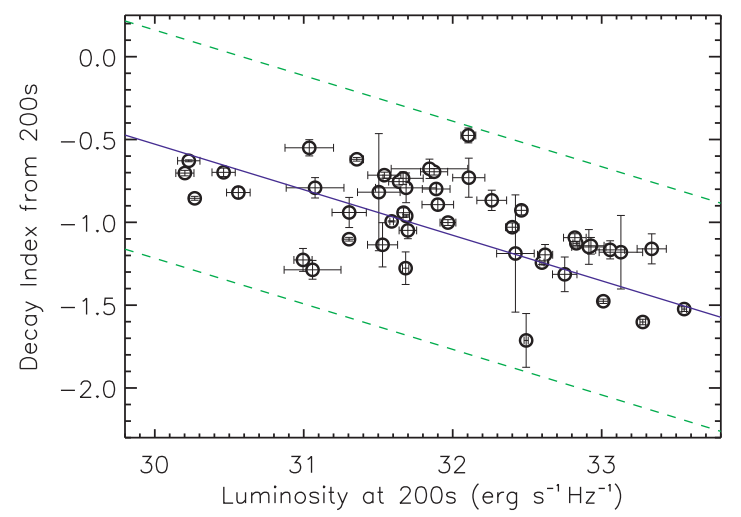

Fig. 2. Average decay index determined from the luminosity light curves after $200 \mathrm{~s}$ versus luminosity at $200 \mathrm{~s}$. The blue solid line represents the best fit regression, $\log \mathrm{L}_{200 \mathrm{~s}}=(-3.636 \pm 0.004) \alpha+(28.08 \pm 0.13)$, and the green dashed lines represents the $3 \sigma$ deviation.

more slowly. To verify this observation, we performed a Spearman Rank test on the the logarithmic luminosity, $\log \mathrm{L}_{200 \mathrm{~s}}$, interpolated at $200 \mathrm{~s}$ using the data between 100 and $2000 \mathrm{~s}$, and the average decay rate $\alpha_{>200 \mathrm{~s}}$, determined by fitting a single power law to the light curves from $200 \mathrm{~s}$ onwards. We chose a restframe time of $200 \mathrm{~s}$ as all the light curves in the sample have observations by this time. For the 48 GRBs, for which we could determine log $\mathrm{L}_{200 \mathrm{~s}}$ and $\alpha_{>200 \mathrm{~s}}$ (see Fig. 2), a Spearman rank test gives a coefficient of -0.58 at a significance of $99.998 \%(4.2 \sigma)$, confirming that luminous optical/UV afterglows decay quicker than less luminous ones.

To exclude the possibility that the correlation results from both parameters being related to redshift, we determined the partial Spearman rank correlation. This measures the degree of correlation between two parameters, excluding the effect of a third (see Kendall \& Stuart 1979). This results in a coefficient of -0.50 with a confidence of $99.97 \%(3.5 \sigma)$, and indicates that the $\mathrm{L}_{200 \mathrm{~s}}-\alpha_{>200 \text { s }}$ correlation is not a result of the implicit correlation between these two parameters and redshift.

We also examined if the correlation was a result of the selection criteria or due to chance by performing a Monte Carlo simulation using $10^{6}$ trials. For each trial, we simulated a random distribution of 48 pairs of $\log \mathrm{L}_{200 \mathrm{~s}}$ and $\alpha_{>200 \mathrm{~s}}$. For each pair, we calculated the observed frame light curve, using the values of $\log \mathrm{L}_{200 \mathrm{~s}}$ and $\alpha_{>200 s}$, and randomized parameters for redshift, extinction and k-correction. If the resulting observed frame light curve did not meet our selection criteria we discarded the $\log \mathrm{L}_{200 \mathrm{~s}}-\alpha_{>200 \mathrm{~s}}$ data point from the simulated distribution and drew a new pair of values until the selection criteria was met. Once 48 valid pairs had been verified, we ran a Spearman Rank correlation on the distribution.

Of the $10^{6}$ trials, only 34 have a correlation coefficient equal to or indicating a stronger correlation than the real $\mathrm{L}_{200 \mathrm{~s}}-\alpha_{>200 \mathrm{~s}}$ distribution. Therefore at $4.1 \sigma$ 
confidence, the $\mathrm{L}_{200 \mathrm{~s}}-\alpha_{>200 \text { s }}$ correlation is not due to our selection criteria or by chance and implies that the $\mathrm{L}_{200 \mathrm{~s}}-\alpha_{>200 \mathrm{~s}}$ correlation is intrinsic to LGRBs.

\section{Discussion}

We shall now examine 3 ways to produce a $\log \mathrm{L}_{200 \mathrm{~s}}$ and $\alpha_{>200 \mathrm{~s}}$ correlation.

\subsection{Basic GRB afterglow model}

A correlation between $\log \mathrm{L}_{200 \mathrm{~s}}$ and $\alpha_{>200 \mathrm{~s}}$ may be a natural result of the jet interacting with the external medium producing synchrotron emission, which results in a relationship $L \propto t^{\alpha} \nu^{\beta}$, where $\beta$ is the spectral index, and, $\alpha$ and $\beta$ are linearly related by the standard set of closure relations (e.g. Zhang et al. 2006). There are two scenarios that could produce the $\log \mathrm{L}_{200 \mathrm{~s}}-\alpha_{>200 \mathrm{~s}}$ correlation. In the simplest scenario, the optical light curves, for all GRBs, are produced from the same spectral regime and therefore are produced from the same spectral segment and have the same density profile. A single closure relation would describe the relationship between $\alpha$ and $\beta$ and we should expect a correlation between these two parameters and a correlation between $\log \mathrm{L}_{200 \text { s }}$ and $\beta$. However, we can exclude this scenario as we do not observe either of these correlations in Figure 3. In the second scenario, we consider the optical afterglows to be produced by multiple spectral regimes. In Figure 3 we display the closure relations for three most likely spectral regimes. In this more complex scenario, we should expect to see clustering of the luminosities around a given closure relation, but again we do not observe this in Figure 3. We thus exclude a basic standard afterglow model as the cause of the correlation.

\subsection{Complex GRB afterglow model}

There may be an additional mechanism or parameter in the standard model that regulates energy release in GRB afterglows, depending on the rate of decay, such that if the energy is released quickly the result is a bright-fast decaying afterglow, while if the energy is released more slowly a fainter-slower decaying afterglow is observed. One possibility could be continued energy injection. For instance, if the central engine does not release its energy all in one go, but releases it over a much longer duration, then we may expect faint - slowly decaying afterglow.

\subsection{Off-axis and structured outflows}

The $\mathrm{L}_{200 \mathrm{~s}}-\alpha_{>200 \mathrm{~s}}$ correlation may instead be a geometric effect resulting from a range in viewing angle, $\theta_{\text {obs }}$. The faint-slowly decaying optical afterglows would be those observed at the largest angles, while the bright-fast decaying light curves would be observed within the outflow (see Fig. 3 of Panaitescu \& Vestrand 2008). If the outflow is also structured, the result is similar, but the convergence time 


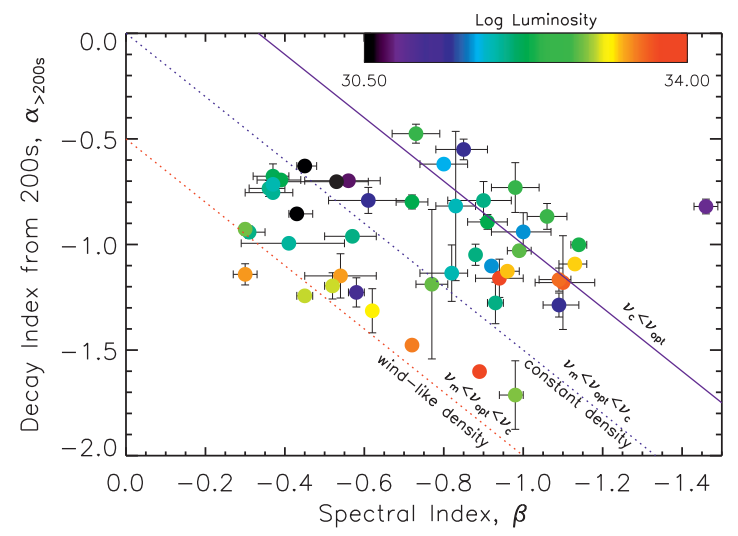

Fig. 3. Optical/UV temporal and spectral indices for the 48 GRBs. The lines represent 3 closure relations and a colour scale is used for the range in luminosity at $200 \mathrm{~s}, \log \mathrm{L}_{200 \mathrm{~s}}$.

and the range of light curve decays will vary, depending on the energy distribution within the jet.

\section{Conclusions}

We find an intrinsic correlation between optical/UV luminosity at $200 \mathrm{~s}$ and average decay rate determined from $200 \mathrm{~s}$ onwards with a significance of $99.998 \%$ $(4.2 \sigma)$. We give two possible causes: an intrinsic mechanism or physical quantity that controls how quickly the energy is released, such that bright, fast-decaying afterglows release their energy more quickly than faint, slow-decaying afterglows; alternatively, the observers viewing angle may produce the correlation, with observers at large viewing angles observing fainter and slower decaying light curves.

This research has made use of data from the High Energy Astrophysics Science Archive Research Center (HEASARC) and the Leicester Database and Archive Service (LEDAS), provided by NASA's Goddard Space Flight Center and the Department of Physics and Astronomy, Leicester University, UK, respectively. SRO, AAB, NPMK, and MJP acknowledge the support of the UK Space Agency.

\section{References}

Kendall, M., \& Stuart, A., 1979, The advanced theory of statistics, Vol. 2, Inference and relationship

Oates, S.R., Page, M.J., Schady, P., et al., 2009, MNRAS, 395, 490

Panaitescu, A., \& Vestrand, W.T., 2008, MNRAS, 387, 497

Roming, P.W.A., Koch, T.S., Oates, S.R., et al., 2013, in preparation

Zhang, B., Fan, Y., Dyks, J., et al., 2006, ApJ, 642, 354 
